\title{
Doped Metasurfaces: Optical Metasurfaces without Etched Structure based on Regular Spatially-doped Semiconductor and Compatible with General Functional Optical Films
}

Ke Jin

Xi'an Institute of Applied Optics

Ying Ding

Sun Yat-Sen University

Lu Cheng

Sun Yat-Sen University

Wei Zheng ( $\square$ zhengw37@mail.sysu.edu.cn)

Sun Yat-Sen University https://orcid.org/0000-0003-4329-0469

Research

Keywords: Doped metasurfaces, modulation of light field, anti-reflection film, without etched

Posted Date: April 3rd, 2021

DOl: https://doi.org/10.21203/rs.3.rs-376961/v1

License: (c) (i) This work is licensed under a Creative Commons Attribution 4.0 International License.

Read Full License 


\title{
Doped Metasurfaces: Optical metasurfaces without
}

\author{
etched structure based on regular spatially-doped
}

semiconductor and compatible with general

\section{functional optical films}

Ke Jin ${ }^{1, \dagger}$, Ying Ding ${ }^{2, \dagger}$, Lu Cheng ${ }^{2}$, Wei Zheng ${ }^{2, *}$

$\dagger$ These authors contributed equally to this work.

${ }^{1}$ Xi' an Institute of Applied Optics, Xi'an 710065, China

${ }^{2}$ State Key Laboratory of Optoelectronic Materials and Technologies, School of Materials, Sun Yat-sen University, Guangzhou 510275, China.

* Corresponding Author (email: zhengw37@mail.sysu.edu.cn)

Abstract: As a new-type artificial two-dimensional planar array structure, metasurfaces can achieve the modulation of light field through a reasonable design and arrangement of subwavelength nano-structure units, which is expected to surpass conventional optical components theoretically. However, the metal or dielectric metasurfaces fabricated by etching process are often trapped in the problems including low focusing efficiency and difficult preparation, which greatly hinders their practical implementation. Here, an idea of "Doped metasurfaces" based on a spatial and regular doping of semiconductor thin films is proposed for the first time. This kind of metasurfaces without etched structure owns smooth and flat surface, allowing the addition of optical functional films like anti-reflection film, which thus gets their optical 
properties greatly improved and enriched on the basis of meta-structured focusing characteristics. In order to verify the effectiveness of this strategy, we simulate a doped metalens suitable for wide mid-infrared (MIR) range and design an anti-reflection film on it. It is shown that this designed MIR metalens possesses wide operating range, high transmittance and high focusing efficiency. The method proposed here provides a new idea or perspective for constructing metasurfaces devices compatible with traditional optical thin films.

Keywords: Doped metasurfaces, modulation of light field, anti-reflection film, without etched.

\section{Introduction}

Metasurfaces, an innovative concept in the fields of optics, has attracted extensive attention [1, 2]. Metasurfaces refer to a two-dimensional functional planar structure composed of numerous sub-wavelength nano-structure units. Through an interaction between the sub-wavelength structure and incident light field, an efficient modulation on amplitude, phase and polarization of electromagnetic (EM) wave can be achieved, which is expected to subvert conventional optical components theoretically [3]. A practical application of metasurfaces is namely metalens, which is known for its characteristics of being ultrathin and flat, as well as the ability to focus light just like ordinary lens $[4,5]$. These performance makes metalens expected to be a revolutionary technology in optics field by replacing the complex and bulky lens group, so as to change mobile phones, cameras and other products smaller and thinner in the future, which explains why this concept could lead a research boom in optics field.

When it comes to the design of metasurfaces, there are three main categories including metallic antenna array metasurfaces $[3,4,6]$, dielectric metasurfaces [7], and metal-dielectric 
hybrid metasurfaces [8]. However, behind all the types listed above lie some specific scientific or technical obstacles which hinder the practical implementation. For example, a strong loss would be resulted from the interaction between EM wave and free electrons of metal, which generally causes low transmission and focusing efficiency of metal-based metasurfaces $[9,10]$. Situation is improved to some extent for dielectric-based metasurfaces whose focusing efficiency is much higher, but it is still limited by a scattering of unit structure and a mismatch between the equivalent admittance of the metasurfaces' surface layer and the incident medium or substrate [11]. In addition, dielectric metasurfaces are usually made up of high refractive index dielectric columns with a length comparable to the wavelength of EM wave as shown in Fig. 1(a), which will lead to a quite difficult preparation and processing process when they are used in mid- or far-infrared range due to the large depth-width radio of dielectric column [1].

Facing those problems, we propose a new design scheme here based on spatial regular doping of semiconductor films: through the technical means like plasma implantation, specific region of the semiconductor thin films can be doped to change the dielectric function of that doped region, after which an array structure similar to that of traditional metasurfaces can be further designed to realize an efficient modulation of EM wave, as shown in Figs. 1(b-c). The doped region unit here is in fact equivalent to the etched nano-structure of conventional metasurfaces, which means a new type of metasurfaces, namely Doped metasurfaces, can be constructed by reasonably designing and arranging the doped units. Compared with traditional etched-structure metasurfaces, doped metasurfaces have unique merits: without etched structure, they have a smooth and flat surface which makes some functional films on it like optical anti-reflection film accessible, as shown in Fig. 1(e). In other words, doped 
metasurfaces can both realize the focusing function owned by normal metasurfaces and stay compatible with functional optical thin films as what traditional lens does, so as to further improve and expand their optical properties.

\section{Results and Discussion}

Based on the design scheme introduced above, we apply Generalized Snell' Law to the doped metasurfaces. Fig. 1(d) shows the schematic of structure and phase changes of doped metasurfaces, where there are two interfaces with one between incident medium and doped layer and the other between doped layer and substrate. The Generalized Snell's Law is applied to these two interfaces, respectively, as follows [3]:

$$
\begin{aligned}
& \sin \left(\theta_{d}\right) n_{d}-\sin \left(\theta_{i}\right) n_{i}=\frac{\lambda_{0}}{2 \pi n_{i}} \nabla \phi_{0} \\
& \sin \left(\theta_{t}\right) n_{t}-\sin \left(\theta_{d}\right) n_{d}=\frac{\lambda_{0}}{2 \pi n_{d}} \nabla \phi_{1}
\end{aligned}
$$

where $\theta_{i}$ is incident angle, $\theta_{d}$ and $\theta_{t}$ the refraction angle of doped layer and substrate, respectively, $n_{i}, n_{d}, n_{t}$ the refractive index of incident medium, doped layer and the substrate, respectively, and $\nabla \phi_{0}$ and $\nabla \phi_{1}$ are the phase gradient generated by the two interfaces of metastructures. By adding equations (1) and (2) together, a Generalized Snell's Law suitable for doped metasurfaces is obtained:

$$
\sin \left(\theta_{t}\right) n_{t}-\sin \left(\theta_{i}\right) n_{i}=\frac{\lambda_{0}}{2 \pi n_{i}} \nabla \phi_{0}+\frac{\lambda_{0}}{2 \pi n_{d}} \nabla \phi_{1}
$$

It can be seen from equation (3) that the phase change introduced by doping metasurfaces is contributed by the upper and lower interfaces, respectively, which has nothing to do with the thickness of doped layer, which means the phase change from 0 to $2 \pi$ can be realized by reasonably designing the phase gradient of the upper and lower interfaces.

In order to verify the correctness of this idea of doped metasurfaces, a metalens based on 
a Sn-doped $\mathrm{In}_{2} \mathrm{O}_{3}$ thin film doped metasurfaces for mid-infrared range is designed and FDTD method is used to stimulate the metalens' optical properties. According to Drude model, the dielectric function $\varepsilon_{m}$ of doped region can be expressed as a function between angular frequency $\omega$ of incident light and free-electron concentration $N_{e}$ [12]:

$$
\varepsilon_{m}=\varepsilon_{R e}+i \varepsilon_{I m}=\varepsilon_{\infty}-\frac{N_{e} q^{2}}{m_{e}\left(\omega^{2}+\gamma_{e}^{2}\right) \varepsilon_{0}}+i \frac{N_{e} q^{2} \gamma_{e}}{m_{e} \omega\left(\omega^{2}+\gamma_{e}^{2}\right) \varepsilon_{0}}
$$

Where $q, \gamma_{e}, \varepsilon_{\infty}, m_{e}$ and $\varepsilon_{0}$ are electron charge, electron damping rate, high frequency dielectric constant $\left(\varepsilon_{\infty}=3.8\right)$, electron effective mass $\left(m_{e}=1.4 m_{0} / \varepsilon_{\infty}\right)$ and vacuum dielectric constant, respectively. The structure and optical properties of doping metasurfaces obtained by simulation are shown in Fig. 2. For details, Fig. 2(a) displays the structure of doped metasurfaces with sillicon as a substrate, where a spatially regularly-arranged Sn-doped $\mathrm{In}_{2} \mathrm{O}_{3}$ thin film with a period of $1 \mu \mathrm{m} * 1 \mu \mathrm{m}$ is formed by the single doped region which is a cuboid whose length, width and thickness of $0.8 \mu \mathrm{m}, 0.2 \mu \mathrm{m}$ and $0.1 \mu \mathrm{m}$ respectively and with a free electron concentration as about $8 \times 10^{20} \mathrm{~cm}^{-3}$ and an azimuthal angle $\varphi$ between the long surface axis and Y axis; Fig. 2(b) shows that when left-handed circularly-polarized light with a wavelength of $4.5 \mu \mathrm{m}$ is incident, the phase change can cover a range from 0 to $2 \pi$ with the change of angle $\varphi$; Fig. 2(c) shows the dielectric function of that doped region in 3-6 $\mu \mathrm{m}$ range. A spherical wavefront is constructed by hyperbolic phase distribution. For normal incident beam with wavelength of $\lambda$, the phase field can be expressed as $\varphi(x, y)=\frac{2 \pi}{\lambda}(f-$ $\sqrt{x^{2}+y^{2}+f^{2}}$, where $f$ is the focal length of lens and $(x, y)$ is the position coordinates of doped unit. Fig. 3 shows the light field distribution of the doped metalens with different thickness $(0.1 \mu \mathrm{m}, 0.2 \mu \mathrm{m})$ of doped layer. It can be seen from the figure that the focal spot size and the intensity of metasurfaces with two thickness are basically the same, which 
indicates that the there is almost no effect of doped area on the loss of light energy when the difference of thickness is little. The focal length of the doped metalens remains $11 \mu \mathrm{m}$ approximately without any change. This situation can be explained by equation (4), which points out that the phase change introduced by doped metasurfaces is only related to the refractive index of two interfaces rather than the thickness of doped layer. In general, the simulation calculation shown above confirms the reliability of doped metasurfaces which are expected to be applied to practical fabrication.

As mentioned before, due to the absence of etched structure, the performance of some functional films such as anti-reflective films can be further improved by being deposited on doped metasurfaces. In order to verify how optical film affects the performance of doped metasurface, we design an anti-reflection film for the substrate in combination with a metasurface layer. The dielectric function of the metasurface is calculated using equivalent medium theory, then the vertical and parallel dielectric functions can be expressed as [13]:

$$
\begin{aligned}
& \varepsilon_{\perp}=\frac{\left(1+f^{\prime}\right) \varepsilon_{m} \varepsilon_{I n_{2} o_{3}}+\left(1-f^{\prime}\right) \varepsilon_{I n_{2} o_{3}}^{2}}{\left(1-f^{\prime}\right) \varepsilon_{m}+\left(1+f^{\prime}\right) \varepsilon_{I n_{2} o_{3}}} \\
& \varepsilon_{\|}=f^{\prime} \varepsilon_{m}+\left(1-f^{\prime}\right) \varepsilon_{I n_{2} o_{3}}
\end{aligned}
$$

where $f^{\prime}$ is the doping duty cycle. $\mathrm{ZnS}$ and $\mathrm{SiO}_{2}$ which are transparent in mid-infrared range and firmly bonded with $\operatorname{In}_{2} \mathrm{O}_{3}$ thin film as well as the substrate are chosen as the anti-reflection film materials. Multi-layer characteristic matrix is used to calculate the anti-reflection film:

$$
\left[\begin{array}{l}
B \\
C
\end{array}\right]=\left\{\prod_{i=1}^{N}\left[\begin{array}{cc}
\cos \delta_{i} & \frac{i}{\eta_{i}} \sin \delta_{i} \\
i \eta_{i} \sin \delta_{i} & \cos \delta_{i}
\end{array}\right]\right\}\left[\begin{array}{c}
1 \\
\eta_{N+1}
\end{array}\right]
$$

where $\delta_{i}$ and $\eta_{i}$ are the phase thickness and material admittance of $i$ layer, respectively, and $\eta_{N+1}$ is the substrate admittance. During the calculation, let $Y=\frac{B}{C}=n_{0}$ be the effective admittance (where $n_{0}$ is air refractive index). Simulation result shows that the anti-reflection 
film should be composed of $0.4 \mu \mathrm{m}$-thick $\mathrm{ZnS}$ and $0.1 \mu$ m-thick $\mathrm{SiO}_{2}$. Fig. 4 compares the light field distributions of doped metasurfaces at different wavelengths $(3.5 \mu \mathrm{m}, 4.5 \mu \mathrm{m}, 5.5 \mu \mathrm{m})$ without and with anti-reflection film. It can be seen that the focal length decreases with the increase of wavelength; under the condition of anti-reflection film, the light intensity at the focus is much higher than that without anti-reflection film, but the focus position is not affected. Fig. 5 is the transmittance curve of 3.0-6.0 $\mu \mathrm{m}$ range and the focusing efficiency is at $3.5 \mu \mathrm{m}$, $4 \mu \mathrm{m}, 4.5 \mu \mathrm{m}, 5 \mu \mathrm{m}$ and $5.5 \mu \mathrm{m}$ wavelengths, from which it can be seen that the transmittance of metalens with anti-reflection film is about $90 \%$ in 3.5-5.5 $\mu \mathrm{m}$ range, which is much higher with an average increase of more than $35 \%$ than that without coating. For focusing efficiency, metalenses with anti-reflection film has a much higher efficiency with an average increase of over $27 \%$ than that without coating, and the highest focusing efficiency can reach $82.2 \%$, which shows that anti-reflection film is a key role in improving the efficiency of metasurfaces.

\section{Conclusions}

Generally speaking, the concept of doped metasurfaces realized by changing dielectric function via doping is put forward for the first time in this paper. The characteristics of doped metalenses in mid-infrared range are studied. Besides, based on the property that doped metasurfaces can be added to functional optical film, the influence of anti-reflection film on transmission and focusing performance of doped metalenses is also studied. Simulation results show that the transmittance and focusing efficiency of doped metalenses with anti-reflection film is much higher than those without this coating. The structure of doped metasurfaces combined with anti-reflection film proposed in this paper is expected to solve the problems including the low infrared efficiency of metal-based metasurfaces and the difficult preparation of dielectric 
metasurfaces. This technology can be applied to the fields of planar lens, vortex phase plate, holographic phase plate, polarization converter and wavelength selector, which brings new perspectives and ideas to the design of metasurfaces devices. 


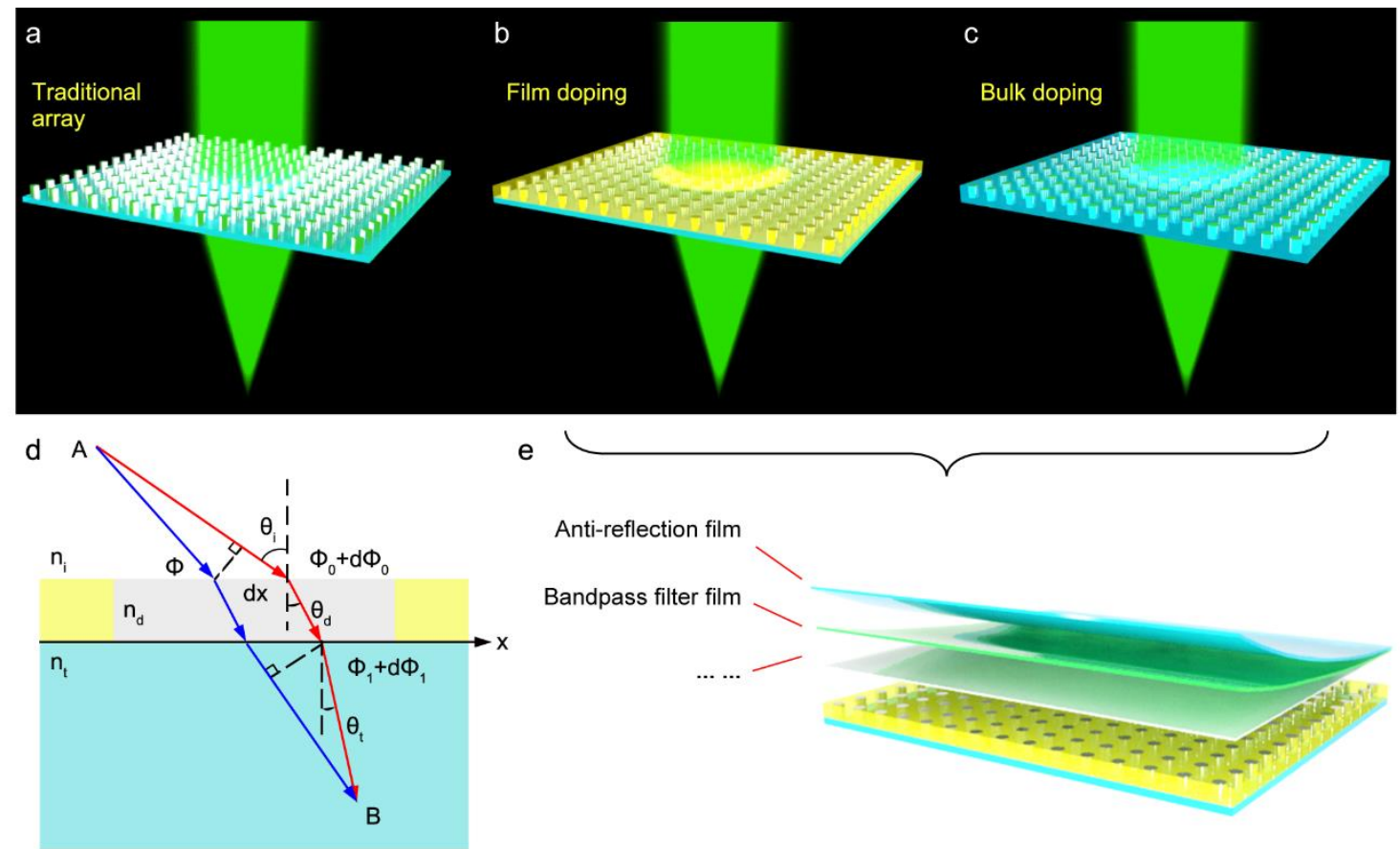

Fig. 1 (a) Traditional metasurface structure. (b) thin-film-doped metasurfaces. (c) doped metamaterial. (d) the schematic of a light wave's introducing a gradient phase mutation through an interface. (e) the schematic of doped metasurface coated with optical film. 
a
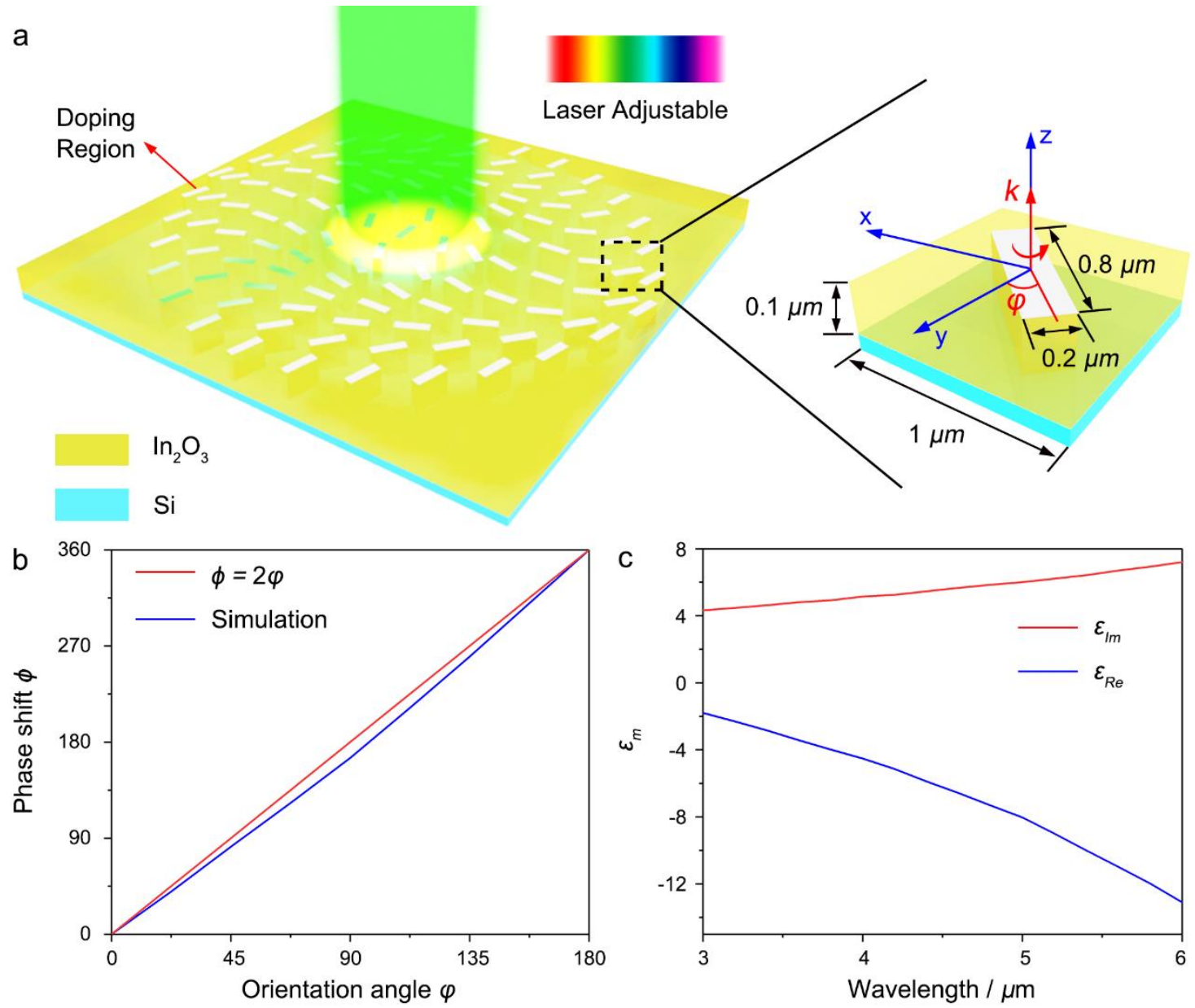

Fig. 2 Design and phase change of doped metasurfaces. (a) doped unit of metasurfaces, which is a cuboid spatially and regularly arranged in $1 \mu \mathrm{m} * 1 \mu \mathrm{m}$ with length, width and thickness as $0.8 \mu \mathrm{m}, 0.2 \mu \mathrm{m}$ and 0.1 $\mu \mathrm{m}$, respectively. (b) phase change as a function of azimuthal angle of the doped unit at a wavelength of 4.5 $\mu \mathrm{m}$, changing from 0 to $2 \pi$. (c) dielectric function of the doped region. 
a

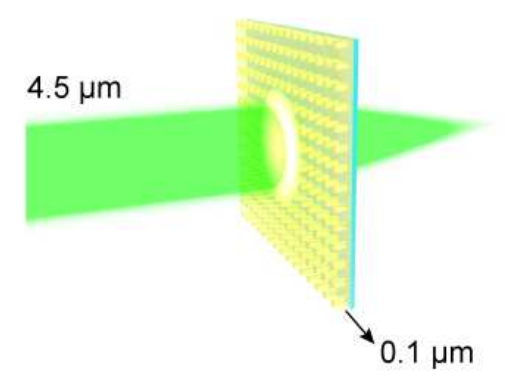

b

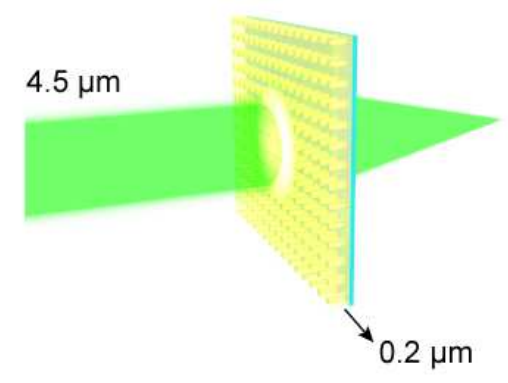

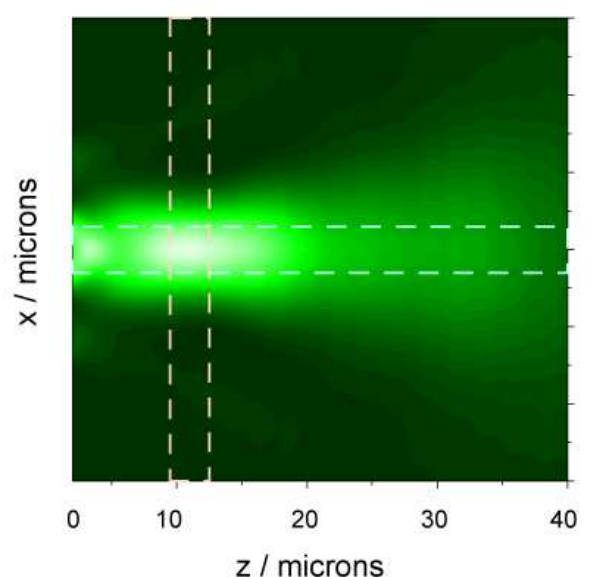
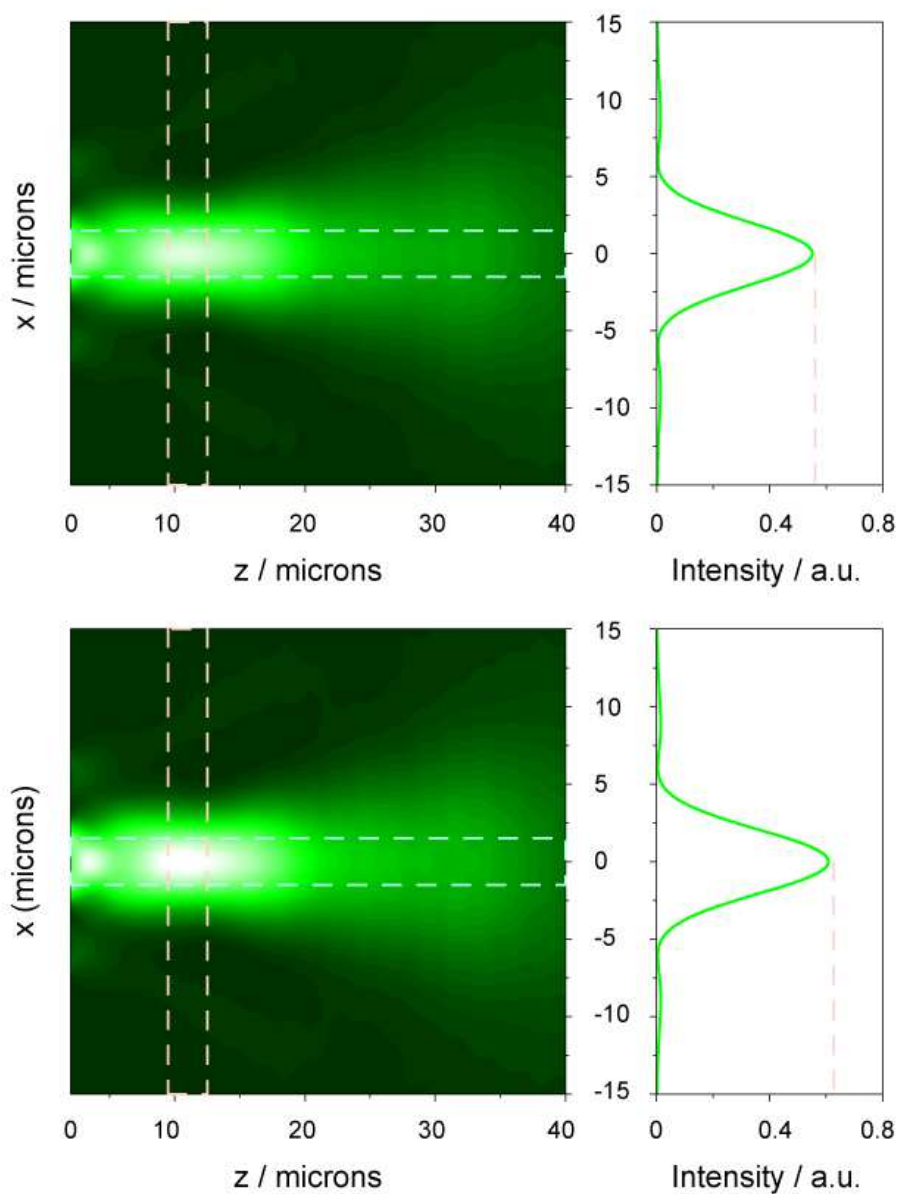

Fig. 3 Light field distribution of focusing lens with different thickness of doped metasurfaces. (a-b) Thickness of the doped layer is $0.1 \mu \mathrm{m}$ and $0.2 \mu \mathrm{m}$, respectively. 
a
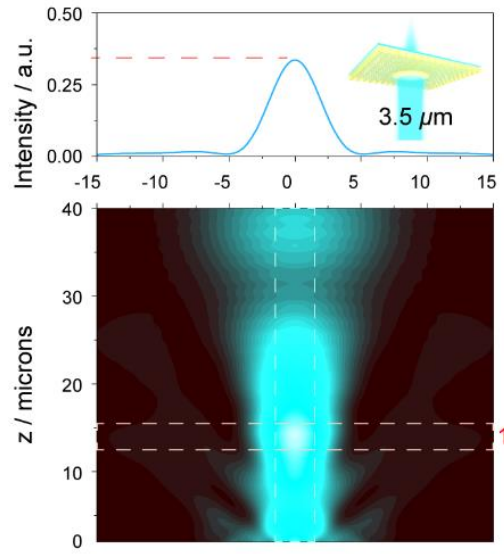

$x /$ microns

b
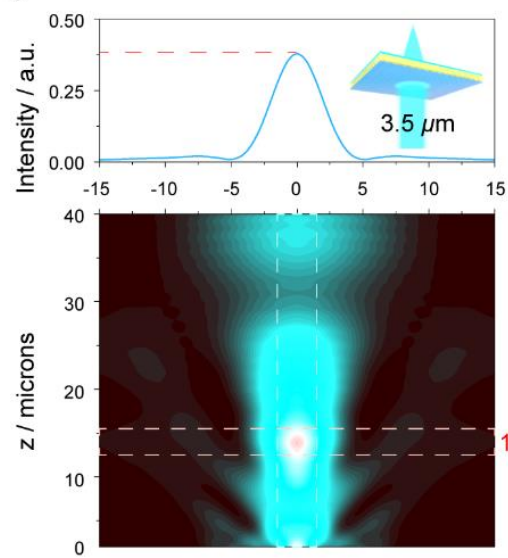

$\mathrm{x} /$ microns
Without Anti-reflection Film
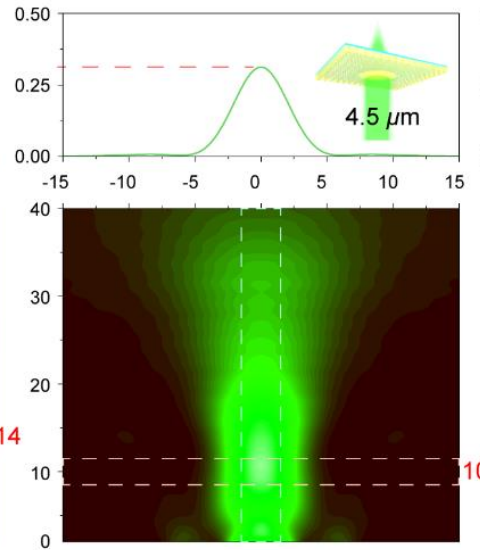

$\mathrm{x} /$ microns
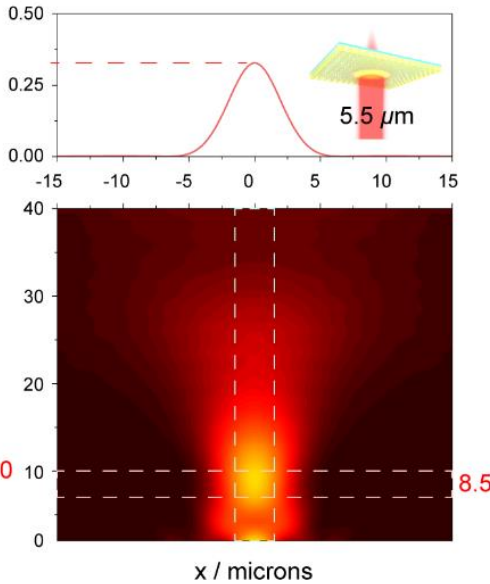

$\mathrm{x} /$ microns
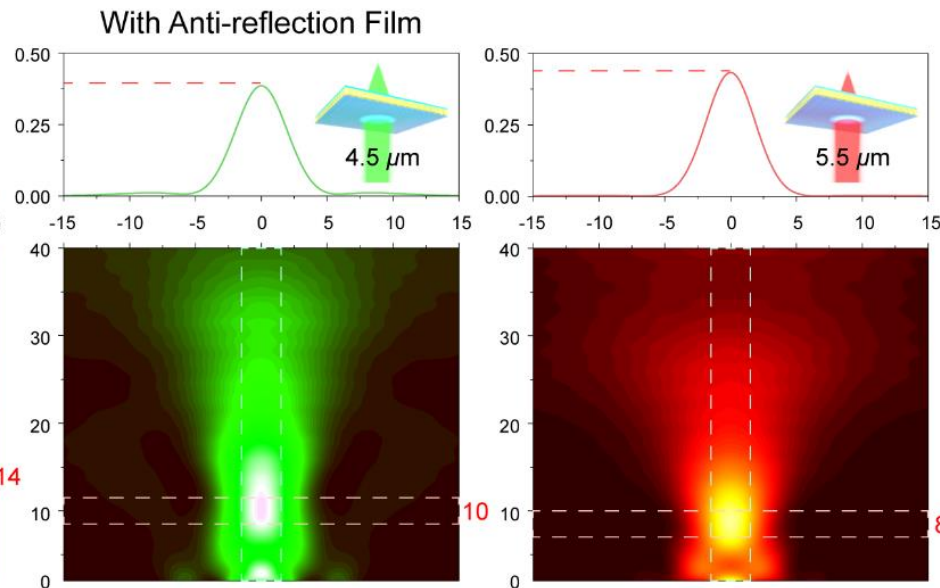

$\mathrm{x} /$ microns

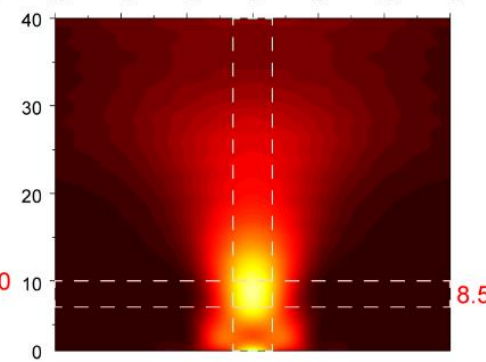

$\mathrm{x} /$ microns

Fig. 4 Light field intensity distribution of the doped metalens at a wavelength of $3.5 \mu \mathrm{m}, 4.5$ $\mu \mathrm{m}$ and $5.5 \mu \mathrm{m}$. (a-c) Without anti-reflection film. (d-f) with anti-reflection film. 

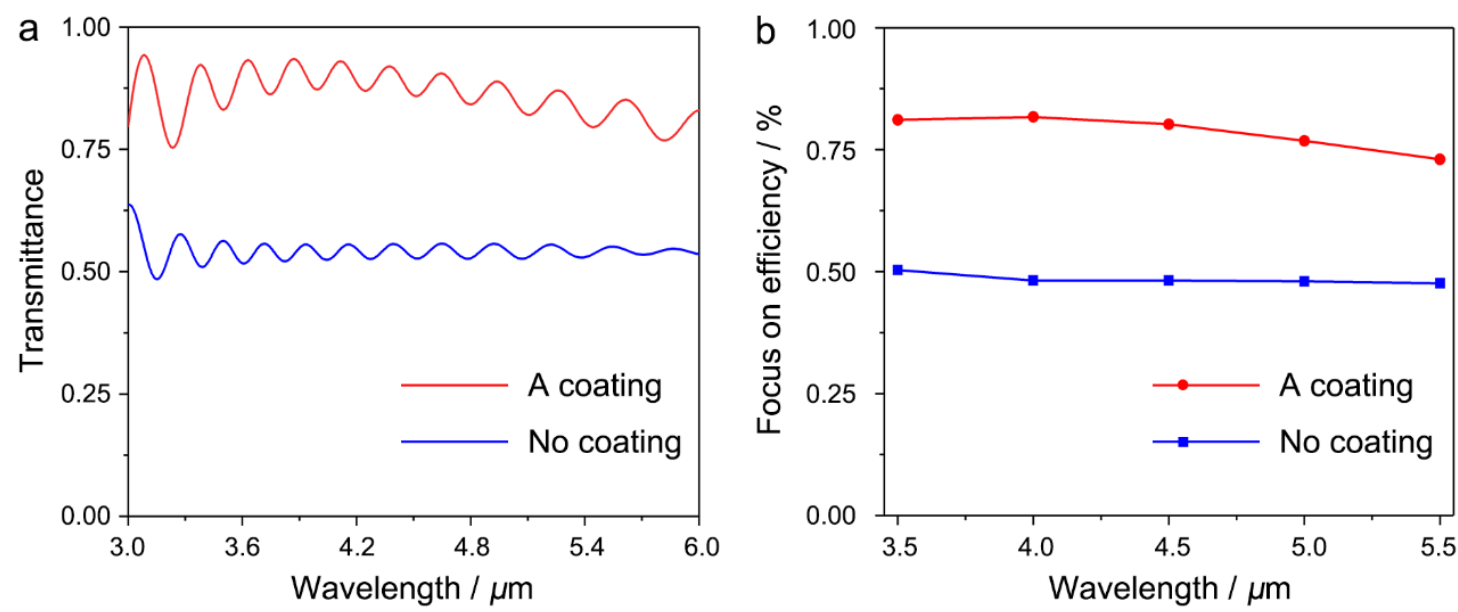

Fig. 5 Comparison of transmittance and focusing efficiency of doped metalens with or without anti-reflection film. (a) Transmittance curve of 3.0-6.0 $\mu \mathrm{m}$ range. (b) focusing efficiency at the wavelength of $3.5 \mu \mathrm{m}, 4 \mu \mathrm{m}, 4.5 \mu \mathrm{m}, 5 \mu \mathrm{m}$ and $5.5 \mu \mathrm{m}$. 


\section{Authors' contributions}

$\mathrm{KJ}$ and $\mathrm{WZ}$ proposed the idea, KJ completed the theoretical calculation, YD, WZ analyzed the data and discussed the results, All the authors wrote the paper and approved the final manuscript.

\section{Availability of data and materials}

The datasets used and analyzed during the current study are available from the corresponding author on reasonable request.

\section{Competing interests}

The authors declare no competing financial interests.

\section{Funding}

There is no funding for the work. 


\section{References}

[1] Q.-B. Fan ,T. Xu, Research progress of imaging technologies based on electromagnetic metasurfaces, Acta Physica Sinica, 66 (2017) https://doi.org/10.7498/aps.66.144208

[2] X. Zou, G. Zheng, Q. Yuan, W. Zang, R. Chen, T. Li, L. Li, S. Wang, Z. Wang ,S. Zhu, Imaging based on metalenses, PhotoniX, 1 (2020) https://doi.org/10.1186/s43074-020$\underline{00007-9}$

[3] N. Yu, P. Genevet, M.A. Kats, F. Aieta, J.P. Tetienne, F. Capasso ,Z. Gaburro, Light propagation with phase discontinuities: generalized laws of reflection and refraction, Science, 334 (2011) 333-7. https://doi.org/10.1126/science.1210713

[4] F. Aieta, P. Genevet, M.A. Kats, N. Yu, R. Blanchard, Z. Gaburro ,F. Capasso, Aberrationfree ultrathin flat lenses and axicons at telecom wavelengths based on plasmonic metasurfaces, Nano Lett, 12 (2012) 4932-6. https://doi.org/10.1021/n1302516v

[5] A.D. Dunkelberger, C.T. Ellis, D.C. Ratchford, A.J. Giles, M. Kim, C.S. Kim, B.T. Spann, I. Vurgaftman, J.G. Tischler, J.P. Long, O.J. Glembocki, J.C. Owrutsky ,J.D. Caldwell, Active tuning of surface phonon polariton resonances via carrier photoinjection, Nature Photonics, 12 (2017) 50-56. https://doi.org/10.1038/s41566-017-0069-0

[6] N.K.E. Xingjie Ni, Alexander V Kildishev, Alexandra Boltasseva, Vladimir M Shalaev, Broadband light bending with plasmonic nanotennas, Science, 335 (2012) https://doi.org/10.1126/science.1214686originally

[7] D. Lin, P. Fan, E. Hasman ,M.L. Brongersma, Dielectric gradient metasurface optical elements, Science, 345 (2014) 298-302. https://doi.org/10.1126/science.1253213

[8] A. Pors ,S.I. Bozhevolnyi, Plasmonic metasurfaces for efficient phase control in reflection, 
Opt Express, 21 (2013) 27438-51. https://doi.org/10.1364/oe.21.027438

[9] S. Sun, Q. He, S. Xiao, Q. Xu, X. Li ,L. Zhou, Gradient-index meta-surfaces as a bridge linking propagating waves and surface waves, Nat Mater, 11 (2012) 426-31. https://doi.org/10.1038/nmat3292

[10] A. Pors, M.G. Nielsen, R.L. Eriksen ,S.I. Bozhevolnyi, Broadband focusing flat mirrors based on plasmonic gradient metasurfaces, Nano Lett, 13 (2013) 829-34. https://doi.org/10.1021/n1304761m

[11] A. Arbabi, Y. Horie, A.J. Ball, M. Bagheri ,A. Faraon, Subwavelength-thick lenses with high numerical apertures and large efficiency based on high-contrast transmitarrays, Nat Commun, 6 (2015) 7069. https://doi.org/10.1038/ncomms8069

[12] Scott H. Brewer ,S. Franzen, Indium Tin Oxide Plasma Frequency Dependence on Sheet Resistance and Surface, J. Phys. Chem. B, 106 (2002) https://doi.org/10.1021/jp026600x

[13] A. Kannegulla ,L.J. Cheng, Subwavelength focusing of terahertz waves in silicon hyperbolic metamaterials, Opt Lett, $41 \quad$ (2016) 3539-42. https://doi.org/10.1364/OL.41.003539 


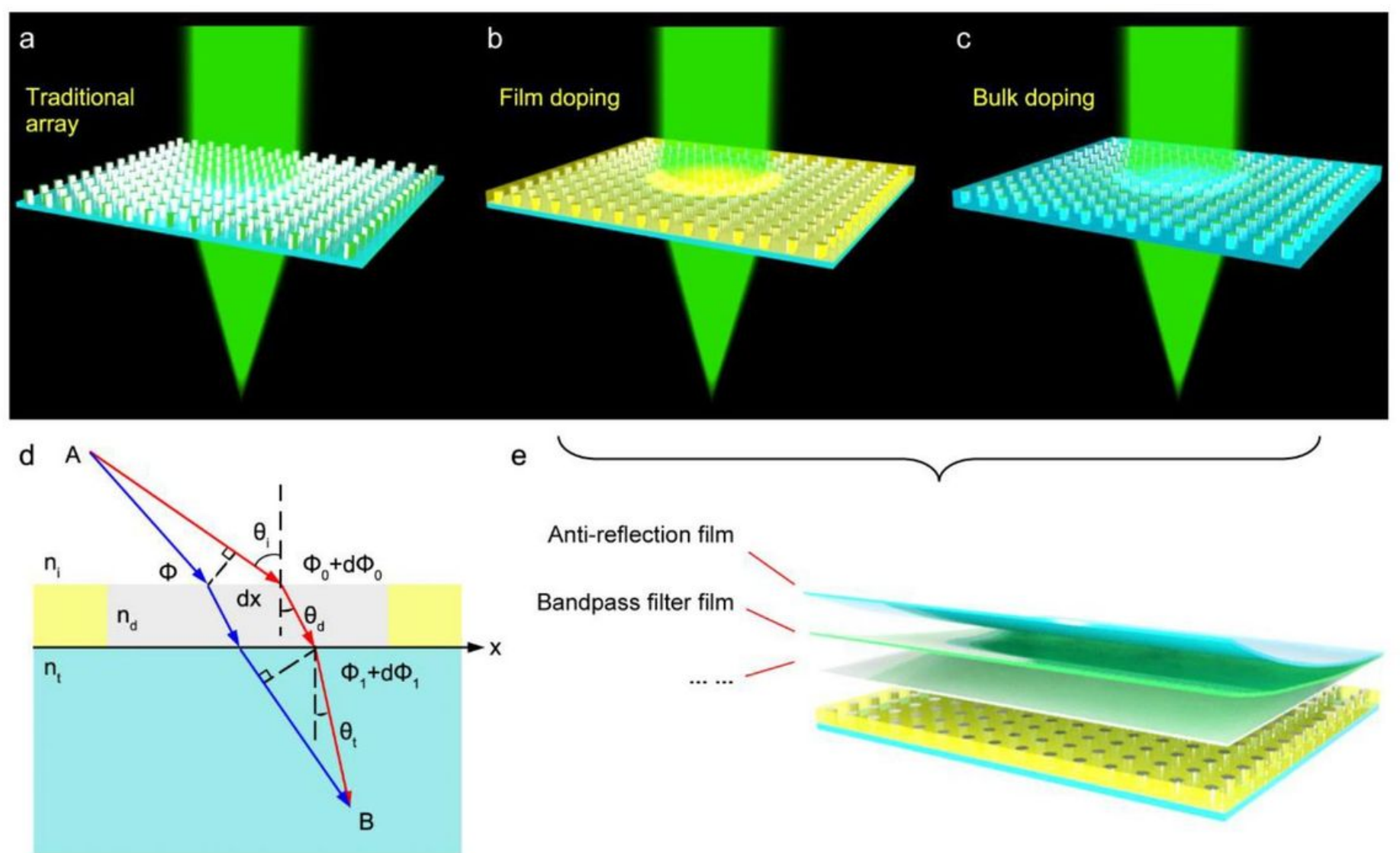

\section{Figure 1}

(a) Traditional metasurface structure. (b) thin-film-doped metasurfaces. (c) doped metamaterial. (d) the schematic of a light wave's introducing a gradient phase mutation through an interface. (e) the schematic of doped metasurface coated with optical film. 

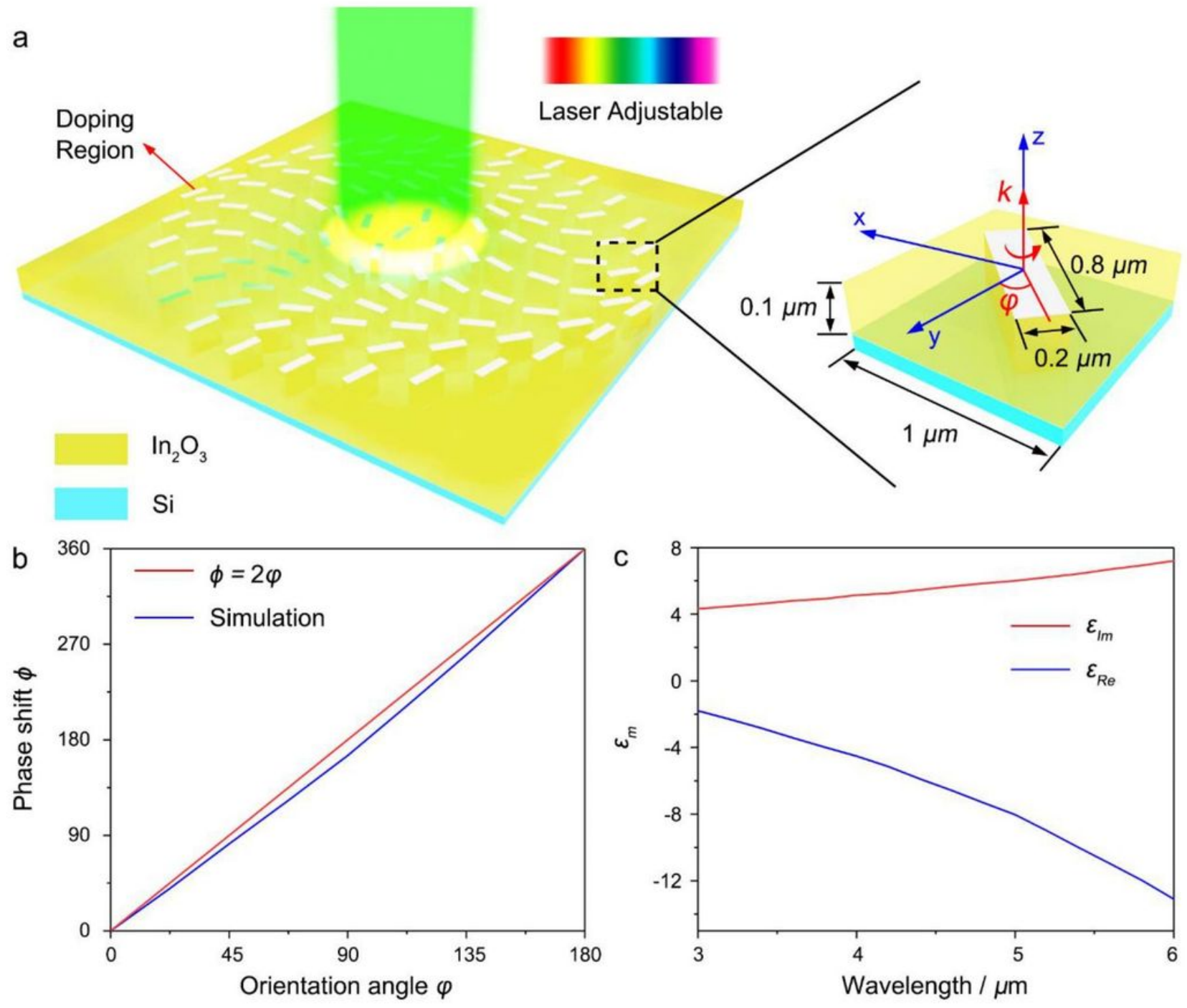

\section{Figure 2}

Design and phase change of doped metasurfaces. (a) doped unit of metasurfaces, which is a cuboid spatially and regularly arranged in $1 \mu \mathrm{m} * 1 \mu \mathrm{m}$ with length, width and thickness as $0.8 \mu \mathrm{m}, 0.2 \mu \mathrm{m}$ and 0.1 $\mu \mathrm{m}$, respectively. (b) phase change as a function of azimuthal angle of the doped unit at a wavelength of $4.5 \mu \mathrm{m}$, changing from 0 to $2 \pi$. (c) dielectric function of the doped region. 
a

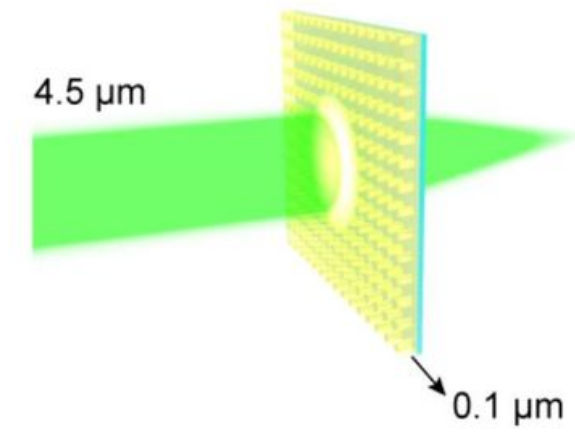

b

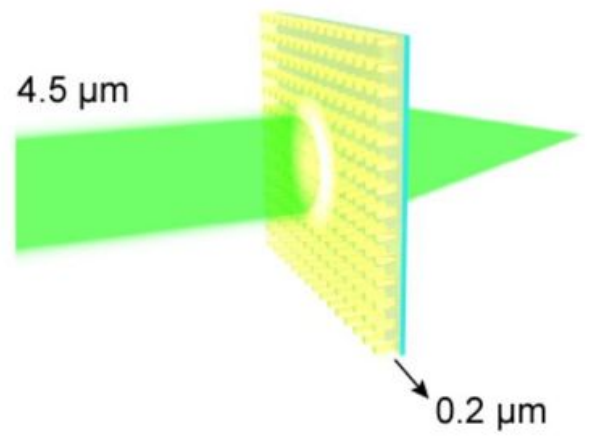

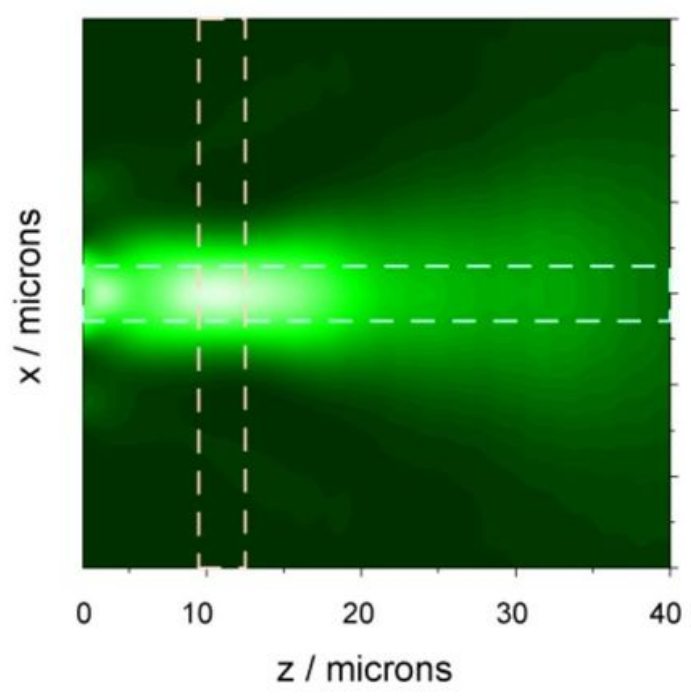
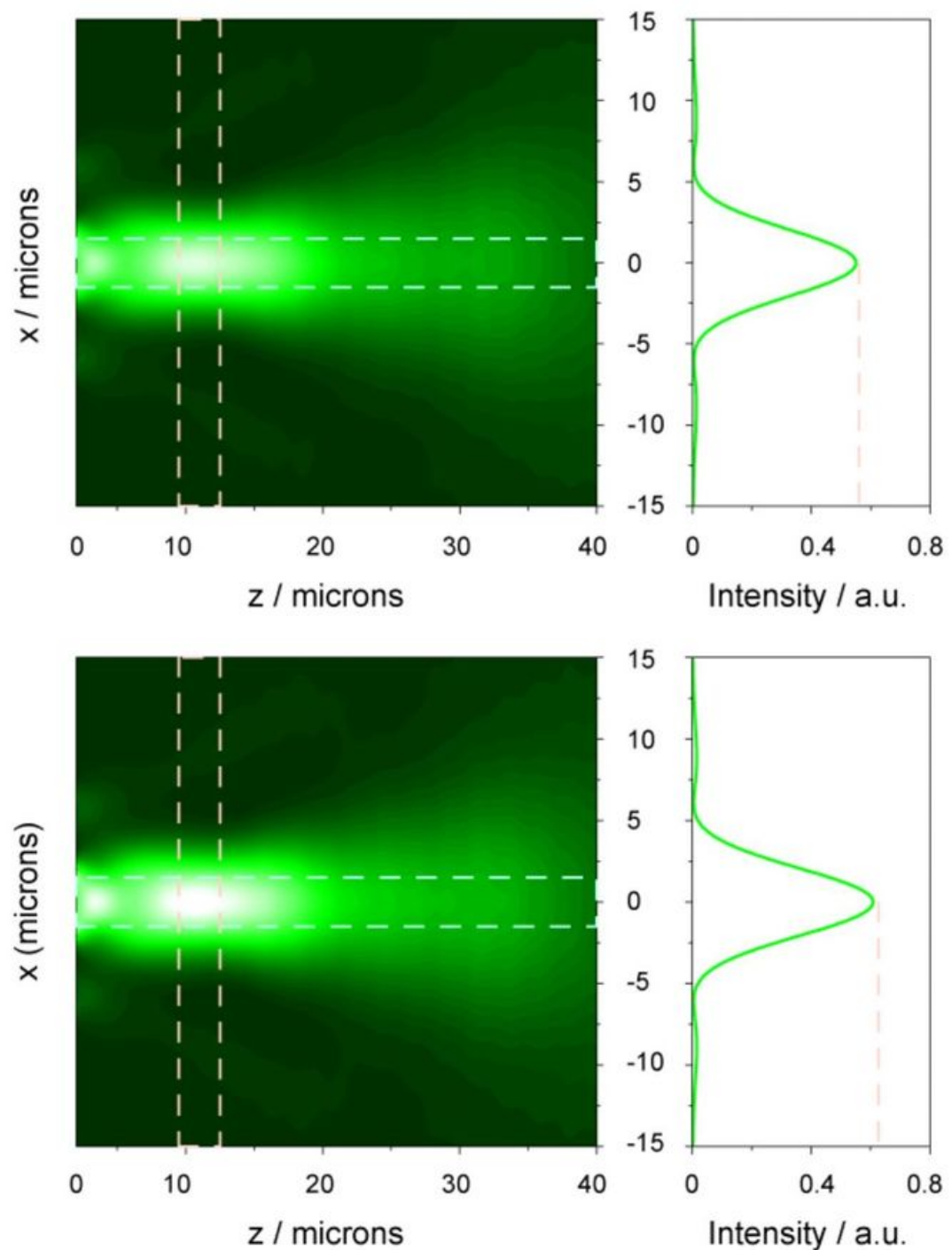

\section{Figure 3}

Light field distribution of focusing lens with different thickness of doped metasurfaces. (a-b) Thickness of the doped layer is $0.1 \mu \mathrm{m}$ and $0.2 \mu \mathrm{m}$, respectively. 
a
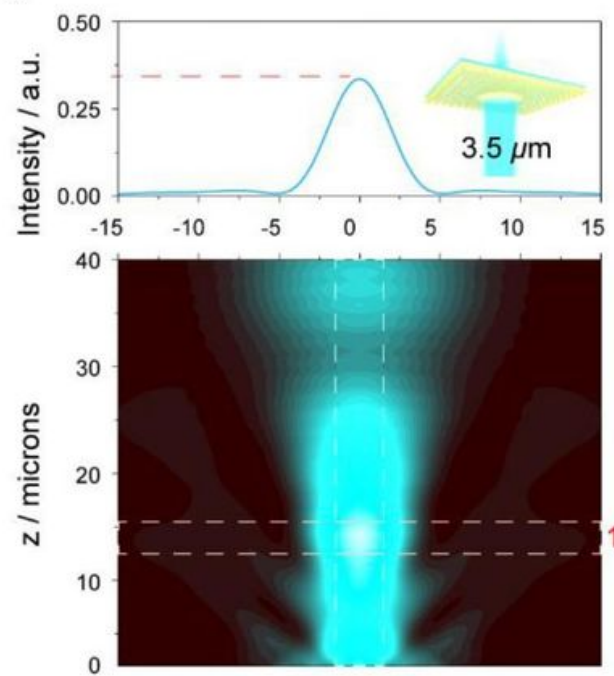

$\mathrm{x} /$ microns

b
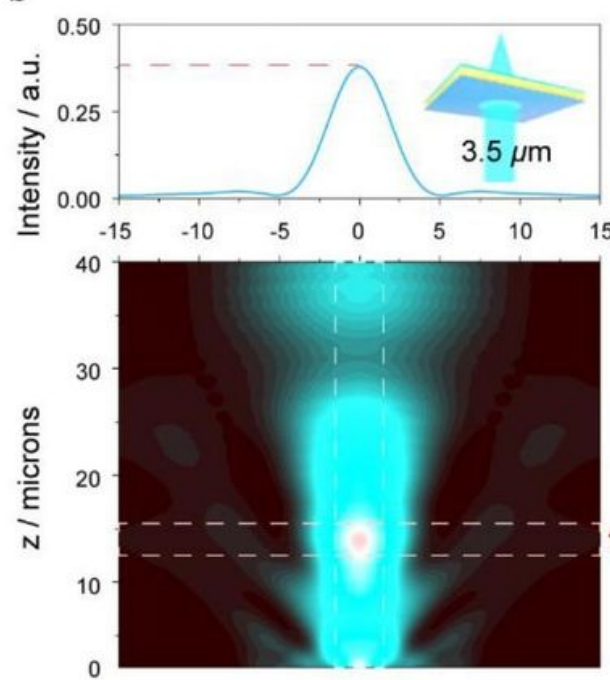

$\mathrm{x} /$ microns
Without Anti-reflection Film
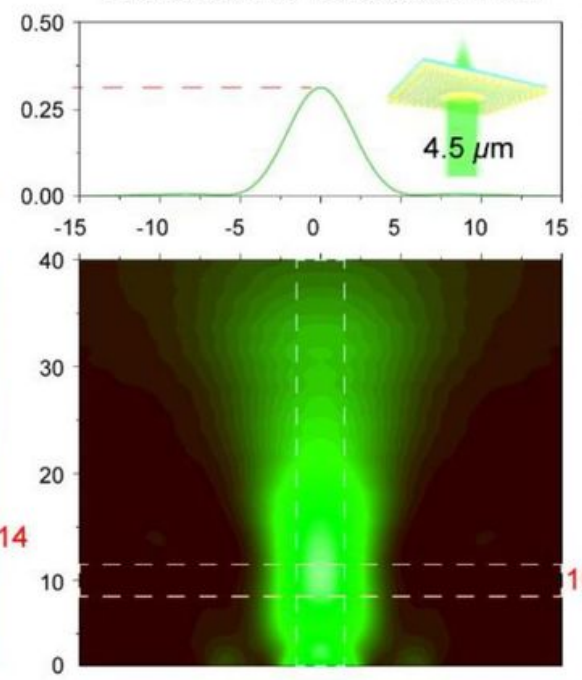

$\mathrm{x} /$ microns
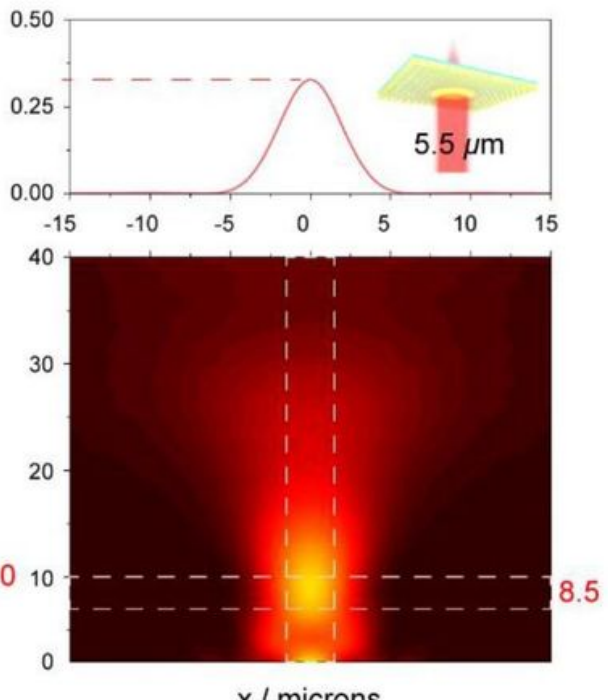

$\mathrm{x} /$ microns

With Anti-reflection Film
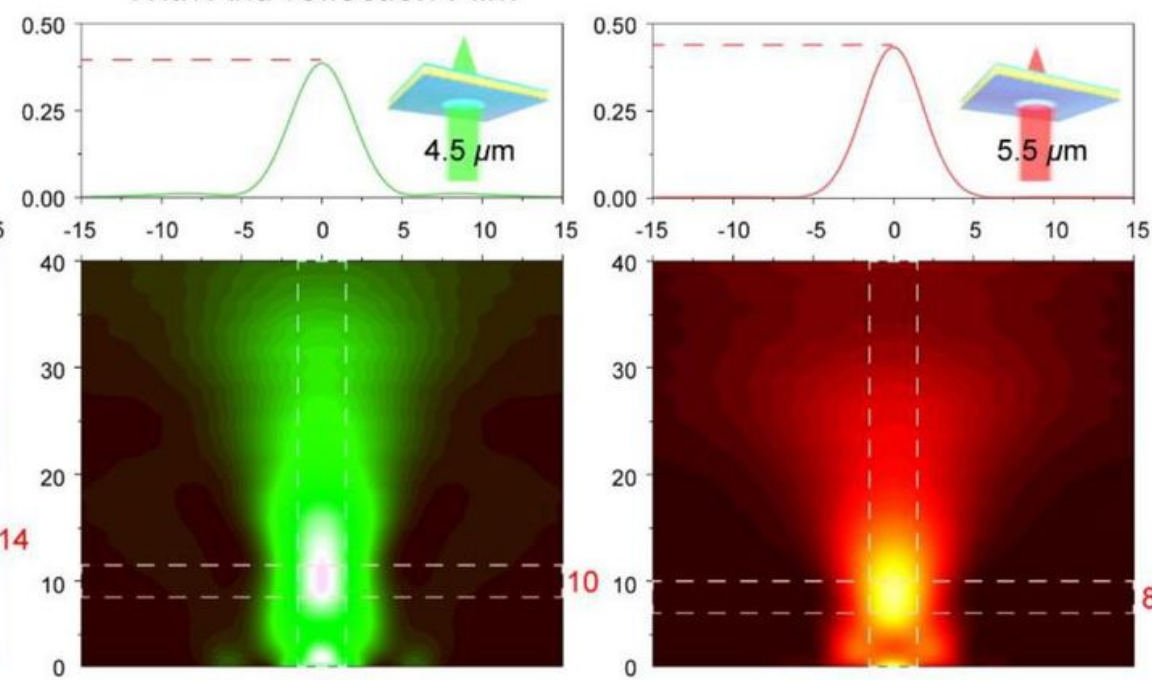

$\mathrm{x} /$ microns

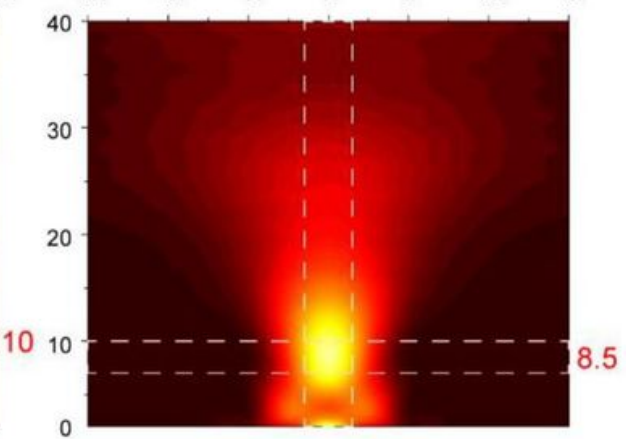

$\mathrm{x} /$ microns

\section{Figure 4}

Light field intensity distribution of the doped metalens at a wavelength of $3.5 \mu \mathrm{m}, 4.5 \mu \mathrm{m}$ and $5.5 \mu \mathrm{m}$. (ac) Without anti-reflection film. (d-f) with anti-reflection film. 

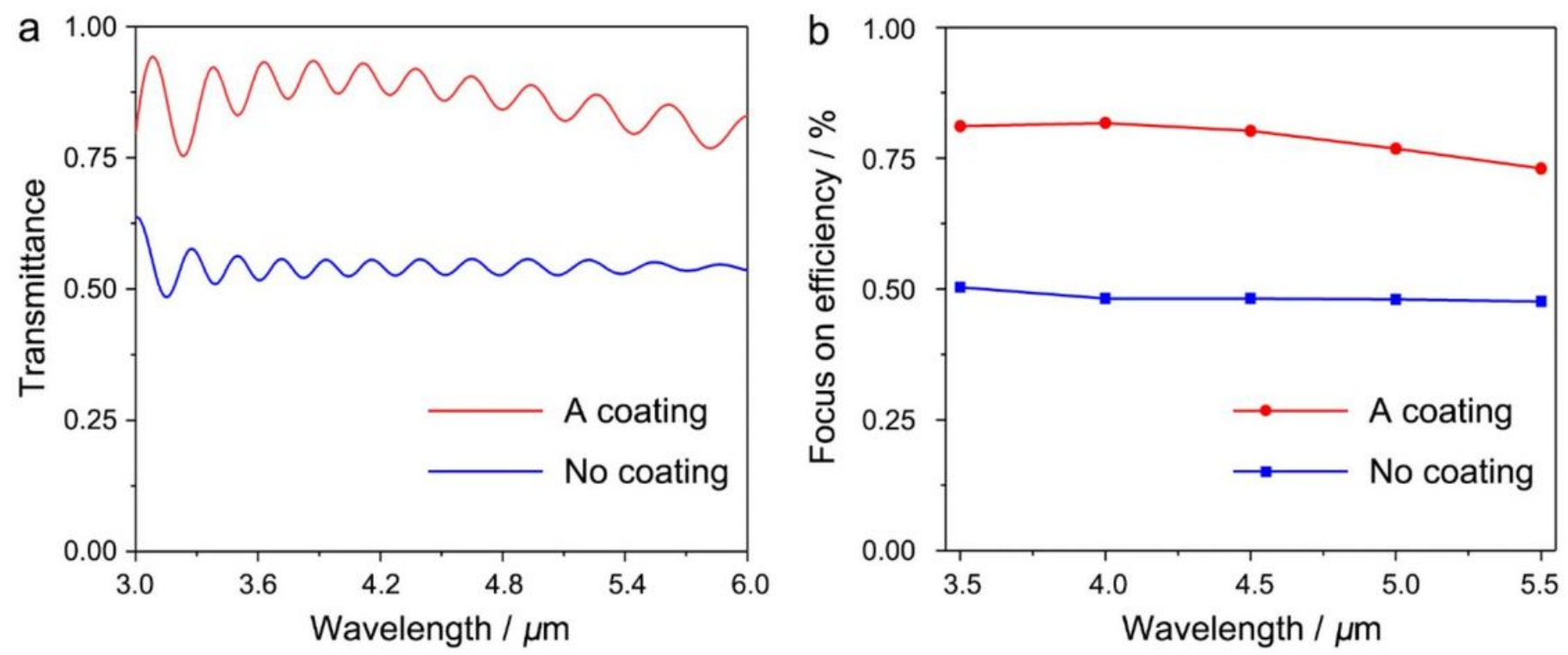

Figure 5

Comparison of transmittance and focusing efficiency of doped metalens with or without anti-reflection film. (a) Transmittance curve of 3.0-6.0 $\mu \mathrm{m}$ range. (b) focusing efficiency at the wavelength of $3.5 \mu \mathrm{m}, 4$ $\mu \mathrm{m}, 4.5 \mu \mathrm{m}, 5 \mu \mathrm{m}$ and $5.5 \mu \mathrm{m}$. 